\title{
Reliability Assessment of Wind Power Growth Planetary Gear Train Based on Dynamics
}

\author{
Chunguang Wang \& Shuai Yue \\ College of mechanical and electrical engineering, Chongqing College of Electric Engineering, Chongqing, \\ China
}

ABSTRACT: From the reliability point of view, the main failure modes of load-split wind power growth planetary gear train are determined by failure mode and effect analysis method. The engineering practice and typical stress and intensity distribution are considered, the probability distribution of random variables are described by the approximate probabilistic method. The wind gear transmission system reliability model is built by the stress-strength interference theory, the failure correlation is analyzed.

KEYWORD: Planetary gear train; Reliability assessment; Dynamics

\section{INTRODUCTION}

Wind turbine is installed in mountains, deserts, beaches and other air outlet, the environment is very harsh. Because of its high altitude, maintenance is difficult, the requirements of reliability and service life is more high than the average mechanical system [1]. Wind power generator gearbox, a low-speed, heavy-duty and speed growth, is a key component in the main drive chain of wind turbine, it can convert wind speed into the required speed in generator [2]. The gearbox failure rate is one of the highest parts in the wind generator [3]. According to statistics, because of failure of gear box, the failure rate of wind turbine rich $12 \%$, the failure probability is about twice as much industrial gear box[4]. So it has important practical significance to analyze and evaluate the reliability of the gear box.

In planetary gear reliability research, Rao researched the reliability of planetary gear transmission system, he thinks the two kinds of failure modes, tooth bending fatigue and pitting, exist in the planetary gear, the change of load is considered, but the research ignores the planet wheel bearing effect[5]. Qing-chun hu[6] and Shangsheng wu[7], based on the system reliability product law and the singlestage planetary gear transmission model proposed by Savage , the reliability model of two-stage planetary gear transmission system is established. Chun-ling li[8] takes 1.5 MW horizontal axis wind wheel gear box sun on as the object of the research, based on dynamic reliability model under random load, the curve of reliability changes with time is built.
In this paper, the load distribution type two-stage planetary and one parallel axis wind gear box is taken on as the research object, the main failure forms of important elements in wind power gear box are determined by the failure mode and effect analysis method.

Combined with the engineering practice and typical distribution of stress and strength, the probability distribution of random variably, such as gear contact stress and strength, bending stress and intensity, is described by the approximate probability method.

\section{SYSTEM DYNAMICS MODEL}

Load-split wind power growth planetary gear train is described as Fig. 1[9].

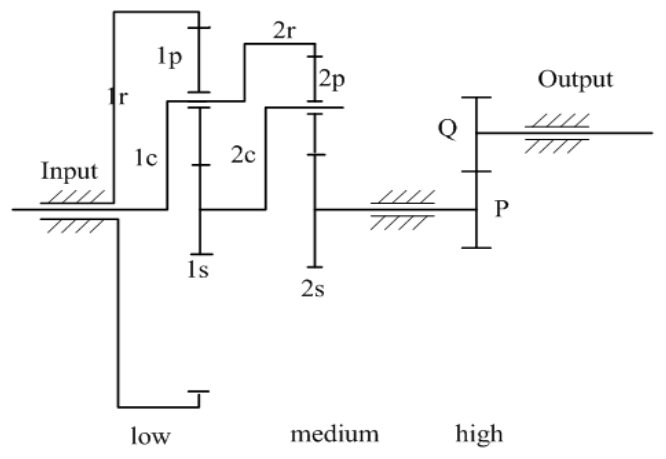

Figure 1. Dynamics model

The system consists of two levels helical planetary gear (low speed level and medium speed level) and one parallel shaft helical gear (high speed level). The 
internal gear ring (1r) of low speed level (noted 1) is fixed, planet (1P) is uniform layout. The planet carrier (1c) of low speed level is connected with the inner gear ring (2r) of medium speed level. The sun (1s) of low speed level is connected with the planet carrier (2c) of medium speed level (noted 2), the planet (2P) of medium speed level is uniform layout. The sun (2s) of medium speed level is connected with helical gear (P) of high speed level. The helical gear of high speed level is connected to the output shaft (P). There are coupling in between low speed level and medium speed level and between medium speed level and high speed level.

To simplified calculation, the sun, planet and ring adopt moving coordinate system, the fixed coordinate which fixed on planet is reference coordinate, it rotates with planet rotation together. For example, the coordinate system of medium speed level is shown in Fig 2, each rotation body contains the movement of the $\mathrm{X}$ and $\mathrm{Y}$ axis and rotation around the $\mathrm{Z}$ axis. Subscript 2c, $2 \mathrm{r}$ and 2 s respectively represent the carrier, ring and the sun of medium speed level. The coordinate system of the Nth planet is $\left(O_{2 n}, X_{2 p n}, Y_{2 p n}, U_{2 p n u}\right)$.

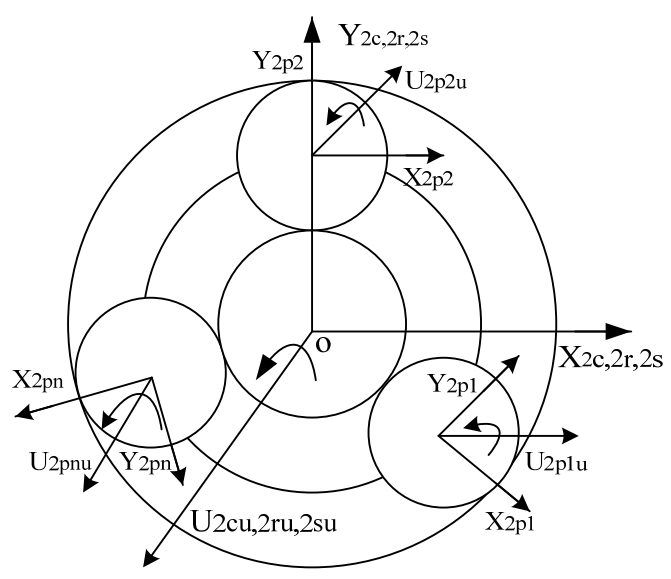

Figure 2. The coordinate system of medium speed level

$\mathrm{X}$ is defined as displacement vector, it includes of meridional displacement and torsional displacement of each gear of low speed, medium speed and high speed level.

Without considering damping, the dynamic equation of the entire transmission system

$M \ddot{X}+K X=T$

In the equation, $\mathrm{K}$ is the system stiffness matrix, $\mathrm{T}$ is external force matrix. According to (1), M, K and $\mathrm{T}$ is derived. Due to limited space, here is not listed in detail.

The elastic deformation along meshing action line between the sun and the nth planet and between the ring and the nth planet are got in medium speed level. The elastic deformation in low speed level and high speed level is similar with medium speed level. The elastic deformation along meshing action line between the sun and the nth planet and between the ring and the nth planet is got in medium speed level. The elastic deformation in low speed level and high speed level is similar with medium speed level.

The elastic deformation $\delta_{2 s 2 \mathrm{pn}}$ along meshing action line between the sun and the nth planet is

$$
\begin{gathered}
\delta_{2 s 2 p n}=y_{2 s} \cos \varphi_{2 s n} \cos \beta_{2}-x_{2 s} \sin \varphi_{2 s n} \cos \beta_{2}-x_{2 p n} \sin \alpha_{2} \cos \beta_{2} \\
-y_{2 p n} \cos \alpha_{2} \cos \beta_{2}+u_{2 s u} \cos \beta_{2}+u_{2 p n u} \cos \beta_{2}
\end{gathered}
$$

The elastic deformation $\delta_{2 r 2 p n}$ along meshing action line between the ring and the nth planet is

$$
\begin{gathered}
\delta_{2 r 2 p n}=y_{2 r} \cos \varphi_{2 r n} \cos \beta_{2}-x_{2 r} \sin \varphi_{2 r n} \cos \beta_{2}-x_{2 p n} \sin \alpha_{2} \cos \beta_{2} \\
-y_{2 p n} \cos \alpha_{2} \cos \beta_{2}+u_{2 r u} \cos \beta_{2}-u_{2 p n u} \cos \beta_{2}
\end{gathered}
$$

In (2) and (3), $\beta_{2}$ is gear spiral angle, $\alpha_{2 s n}$ and $\alpha_{2 r n}$ is meshing pressure angle, $\varphi_{n}$ is the planet position angle. When gear rotate counterclockwise, $\varphi_{2 s n}=\varphi_{n}-\alpha_{2 s n}, \varphi_{2 r n}=\varphi_{n}+\alpha_{2 r n}$.

\section{DYNAMIC MESHING FORCE AND COEFFICIENT}

\subsection{Dynamic Meshing Force}

The dynamic meshing force $P_{2 s p n}$ in between the sun and the nth planet of mediate speed level is

$P_{2 s p n}=K_{2 s p n}(\mathrm{t}) \delta_{2 s p n}$

The dynamic meshing force $P_{2 r n n}$ in between the ring and the Nth planet of medium speed level is

$P_{2 r p n}=K_{2 r p n}(\mathrm{t}) \delta_{2 r p n}$

$K_{2 s p n}$ and $K_{2 \text { rpn }}$ are respectively time-varying mesh force between the sun and the nth planet and between the ring and the Nth planet.

According to the gear instantaneous contact line, time-varying meshing stiffness $K(t)$ is derived [10].

$K(t)=K_{0} L(\tau)$

The average mesh stiffness $K_{0}$ is calculated as follow [11].

$K_{0}=\left(0.75 \varepsilon_{a}+0.25\right) / q$

$q=0.04723+\frac{0.15551}{z_{1}}+\frac{0.25791}{z_{2}}$

The two mutually meshing gear teeth number is $z_{1}$ and $z_{2}$, the symbols "+" means external gear, the symbols "-" means internal gear[12]. Transverse contact ratio

$\varepsilon_{a}=\left[1.88-3.2\left(\frac{1}{z_{1}} \pm \frac{1}{z_{2}}\right)\right] \cos \beta$. 
$L(\tau)=\left[1+\sum_{k=1}^{5}\left(A_{k} \cos (2 \pi k \tau-\varphi)+B_{k} \sin (2 \pi k \tau-\varphi)\right)\right] L_{m}$

In (9), $\varepsilon_{b}$ is gear contact ratio, $\beta_{b}$ is gear base spiral angle, $b$ is helical gear tooth width.

$$
\begin{aligned}
& A_{k}=\frac{1}{2 \varepsilon_{a} \varepsilon_{\beta} \pi^{2} k^{2}}\left[\cos \left(2 \pi k \varepsilon_{\beta}\right)+\cos \left(2 \pi k \varepsilon_{a}\right)-\cos \left(2 \pi k\left(\varepsilon_{a}+\varepsilon_{\chi}\right)\right)\right] \\
& B_{k}=\frac{1}{2 \varepsilon_{a} \varepsilon_{\beta} \pi^{2} k^{2}}\left[\sin \left(2 \pi k \varepsilon_{\beta}\right)+\sin \left(2 \pi k \varepsilon_{a}\right)-\sin \left(2 \pi k\left(\varepsilon_{a}+\varepsilon_{\chi}\right)\right)\right] \\
& L_{m}=b \varepsilon_{a} / \cos \beta_{b}
\end{aligned}
$$

\subsection{Use coefficient and Dynamic Load Coefficient Calculation}

The dynamic meshing force is obtained along the common tangent direction of the two meshing gear base circle, thus the biggest dynamic tangential force $F_{t}^{\prime}$ on gear face graduation circle is obtained.

$$
F_{t}^{\prime}=\max F_{2 j p n} \cos ^{2} \alpha \quad(j=s, r)
$$

The dynamic load coefficient and use coefficient is obtained as follow.

$$
K_{A} K_{V}=\frac{F_{t}^{\prime}}{F_{t}}
$$

In (11), $K_{A}$ is use coefficient, $K_{V}$ is dynamic load coefficient, $F_{t}$ is name tangential force in gear face pitch circle.

\section{RELIABILITY ANALYSIS}

The main failure mode of gear in the wind turbine gear box include gear tooth contact fatigue pitting and tooth root bending fatigue fracture. The rolling bearings main failure mode is pitting corrosion damage in rolling body and inner and outer ring. The input and output shaft, carrier, planet shaft and spline connection have high reliability, so they are ignored in failure mode.

Defined the gear box belongs to a typical cascade system, when there is failure on planets, the planetary gear transmission system is seen as failure. The reliability of the planetary gear transmission system can be considered by the sun, planet, ring and planet bearing at low speed level and medium speed level, and the gear and bearing in high speed level. This series system model is shown in Fig. 3.

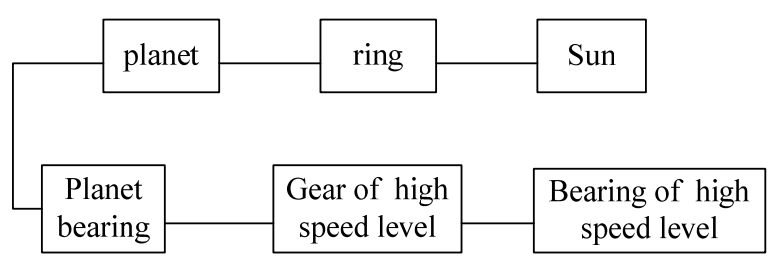

Figure 3. The series system model

\subsection{Reliability calculation}

1) Gear contact strength distribution regularity

(a) Gear tooth surface contact stress calculation model

The contact stress of gear node can be represented as [13]

$$
\sigma_{H}=Z_{H} Z_{E} Z_{\varepsilon} Z_{\beta} \sqrt{\frac{F_{\mathrm{t}}}{\mathrm{d}_{1} b} \frac{u \pm 1}{u} K_{A} K_{V} K_{H \beta} K_{H \alpha}}
$$

In (12), $\sigma_{H}$ is gear contact stress, $Z_{H}$ is node area coefficient , $Z_{E}$ is elastic coefficient, $Z_{\varepsilon}$ is contact ratio coefficient, $Z_{\beta}$ is spiral Angle, $d_{1}$ is pitch circle diameter of small gear , $b$ is work tooth width , $u$ is gear ratio, $K_{A}$ is using factor, $K_{V}$ is dynamic load coefficient, $K_{H \beta}$ is tooth load distribution coefficient, $K_{H \alpha}$ is load distribution coefficient among gears.

(b) Gear tooth surface contact stress distribution model

Tooth surface contact stress is approximate normal probability distribution, the mean and variation coefficient of the gear tooth surface contact stress distribution is shown by second order moment method. For low and medium speed level of planetary gear, the mean and coefficient of variation are shown as follows.

$$
\bar{\sigma}_{H}=Z_{H} \bar{Z}_{E} Z_{\varepsilon} Z_{\beta} \sqrt{\frac{\bar{K}_{C} \bar{F}_{t}}{\mathrm{~d}_{1} b} \frac{u \pm 1}{u} \bar{K}_{A} \bar{K}_{V} \bar{K}_{H \beta} \bar{K}_{H \alpha}}
$$

$C_{\sigma_{H}}=\left[C_{H M}^{2}+C_{Z}^{2}+\frac{1}{4}\left(C_{K_{A}}^{2}+C_{K_{V}}^{2}+C_{K_{H \beta}}^{2}+C_{K_{H \alpha}}^{2}+C_{F_{t}}^{2}+C_{K_{C}}^{2}\right)\right]^{\frac{1}{2}}$

For high speed level of planetary gear, the mean and coefficient of variation are shown as follow.

$$
\begin{aligned}
& \bar{\sigma}_{H}=Z_{H} \bar{Z}_{E} Z_{\varepsilon} Z_{\beta} \sqrt{\frac{\bar{F}_{\mathrm{t}}}{\mathrm{d}_{1} b} \frac{u \pm 1}{u} \bar{K}_{A} \bar{K}_{V} \bar{K}_{H \beta} \bar{K}_{H \alpha}} \\
& C_{\sigma_{H}}=\left[C_{H M}^{2}+C_{z}^{2}+\frac{1}{4}\left(C_{K_{A}}^{2}+C_{K_{V}}^{2}+C_{K_{H \beta}}^{2}+C_{K_{H \alpha}}^{2}+C_{F_{t}}^{2}\right)\right]^{\frac{1}{2}}
\end{aligned}
$$

Pitch circle diameter, tooth width, node area coefficient ,contact ratio coefficient, spiral angle coefficient and so on are related with the gear geometry parameters, they can only change within the allowed precision grade tolerance range, and can be ensured on the process. As a result, they are treated certain variables, the variation coefficient of them should be 0 . In addition to these parameters, other parameters are regard as random variable. $C_{н м}$ is model variation coefficient, it can make sure $\sigma_{H}$ to obey normal distribution.

According to the characteristics of wind turbine gear transmission system, and the variation coeffi- 
cient of $K_{V} 、 K_{H \beta}$ and $K_{H \alpha}$ are respectively $C_{K_{V}}$, $C_{\text {нК } \beta}$ and $C_{\text {нК } \alpha}$.

$C_{K_{V}}=\frac{\bar{K}_{V}-1}{3 \bar{K}_{V}}$

$C_{K_{H \beta}}=\frac{\bar{K}_{H \beta}-1.25}{6 \bar{K}_{H \beta}}$

$C_{K_{H \alpha}}=\frac{\bar{K}_{H \alpha}-1.25}{6 \bar{K}_{H \alpha}}$

Other variation coefficient is got by the characteristics of wind turbine gear transmission system.

The contact stress standard deviation is calculated by

$S_{\sigma_{H}}=C_{\sigma_{H}} \bar{\sigma}_{H}$

The tooth face contact stress can be thought to obey the normal distribution.

$\sigma_{H} \backsim N\left(\bar{\sigma}_{H}, S_{\sigma_{H}}\right)$

(c)The contact fatigue strength calculation model

The contact fatigue strength calculation model is shown as follow.

$\sigma_{H G}=\sigma_{H \lim } Z_{N T} Z_{L} Z_{V} Z_{R} Z_{W} Z_{X}$

In (22),$\sigma_{H G}$ is gear contact limit stress , $\sigma_{H \text { lim }}$ is contact fatigue limit, $Z_{N T}$ is life coefficient of the contact strength , $Z_{L}$ is lubricant coefficient, $Z_{V}$ is velocity coefficient, $Z_{R}$ is roughness coefficient, $Z_{W}$ is work hardening coefficient, $Z_{X}$ is size coefficient .

It is defined that the gear contact fatigue strength obeys lognormal distribution, the average and variation coefficient are respectively got as follow.

$\bar{\sigma}_{H G}=\bar{\sigma}_{H \lim } \bar{Z}_{N T} \bar{Z}_{L} \bar{Z}_{V} \bar{Z}_{R} \bar{Z}_{W} \bar{Z}_{X}$

$C_{\sigma_{H G}}=\left(C_{\sigma_{H} \lim }^{2}+C_{Z_{N T}}^{2}+C_{Z_{L}}^{2}+C_{Z_{V}}^{2}+C_{Z_{R}}^{2}+C_{Z_{W}}^{2}+C_{Z_{X}}^{2}\right)^{\frac{1}{2}}$

$\bar{\sigma}_{H \lim }=\sigma_{b} /\left(1-2.326 C_{\sigma_{H} \lim }\right)$

The contact fatigue strength of logarithmic mean and logarithmic standard deviation can be calculated as follows.

$$
\begin{aligned}
& u_{I n \sigma_{H G}}=I n \bar{\sigma}_{H G}-\frac{1}{2} S_{I n \sigma_{H G}}^{2} \\
& S_{I n \sigma_{H G}}=I n\left(1+\frac{S_{\sigma_{H G}}}{\bar{\sigma}_{H G}^{2}}\right) \\
& S_{\sigma_{H G}}=C_{\sigma_{H G}} \bar{\sigma}_{H G}
\end{aligned}
$$

The tooth surface contact fatigue strength can be thought to obey logarithmic normal distribution, namely
$\operatorname{In} \sigma_{H G} \backsim N\left(u_{I n \sigma_{H G}}, S_{I n \sigma_{H G}}\right)$

2) Tooth root bending stress distribution

(a) The tooth root bending stress calculation mechanical model

The tooth root bending stress calculation mechanical model is shown as follows.

$\sigma_{F}=\frac{F_{t} K_{F \alpha} K_{F \beta} K_{V} K_{A}}{b m_{n}} Y_{F \alpha} Y_{S \alpha} Y_{\varepsilon} Y_{\beta}$

In (30), $\sigma_{F}$ is normal module, $K_{F \beta}$ is tooth coefficient when load acts on the addendum, $K_{F \alpha}$ is stress modification coefficient when load acts on the addendum, $m_{n}$ is normal module, $Y_{F \beta}$ is contact ratio coefficient through the bending strength calculation, $Y_{\beta}$ is spiral angle coefficient.

(b) The tooth root bending stress distribution model

Tooth root bending stress is approximate normal probability distribution, logarithmic mean and logarithmic standard are calculated as follows.

$u_{\text {In } \sigma_{F}}=\operatorname{In} \bar{\sigma}_{F}-\frac{1}{2} S_{\operatorname{In} \sigma_{F}}^{2}$

$\dot{S}_{I n \sigma_{F}}=\operatorname{In}\left(1+\frac{S_{\sigma_{F}}}{\bar{\sigma}_{F}^{2}}\right)$

$S_{\sigma_{F}}=C_{\sigma_{F}} \bar{\sigma}_{F}$

For low and medium speed level

$\bar{\sigma}_{F}=\frac{\bar{F}_{t} \bar{K}_{F \alpha} \bar{K}_{F \beta} \bar{K}_{V} \bar{K}_{A} \bar{K}_{C}}{b m_{n}} \bar{Y}_{F \alpha} \bar{Y}_{S \alpha} Y_{\varepsilon} Y_{\beta}$

$C_{\sigma_{F}}=\left(\begin{array}{l}C_{K_{A}}^{2}+C_{K_{V}}^{2}+C_{K_{F \alpha}}^{2}+C_{K_{F \beta}}^{2}+C_{Y_{\varepsilon}}^{2}+ \\ C_{Y_{\beta}}^{2}+C_{Y_{F \alpha}}^{2}+C_{Y_{S \alpha}}^{2}+C_{F_{\mathrm{t}}}^{2}+C_{K_{C}}^{2}\end{array}\right)$

For high speed level

$\bar{\sigma}_{F}=\frac{\bar{F}_{t} \bar{K}_{F \alpha} \bar{K}_{F \beta} \bar{K}_{V} \bar{K}_{A}}{b m_{n}} \bar{Y}_{F \alpha} \bar{Y}_{S \alpha} Y_{\varepsilon} Y_{\beta}$

$C_{\sigma_{F}}=\left(C_{K_{A}}^{2}+C_{K_{V}}^{2}+C_{K_{F \alpha}}^{2}+C_{K_{F \beta}}^{2}+C_{Y_{\varepsilon}}^{2}+C_{Y_{\beta}}^{2}+C_{Y_{F \alpha}}^{2}+C_{Y_{S \alpha}}^{2}+C_{F_{i}}^{2}\right)$

According to the characteristics of wind turbine gear transmission system, the variation coefficient of $C_{K_{F \beta}}$ and $C_{K_{F \varepsilon}}$ are given as follows.

$C_{K_{F \beta}}=\frac{\bar{K}_{F \beta}-1.2}{6 \bar{K}_{F \beta}}$

$C_{K_{F \alpha}}=\frac{\bar{K}_{F \alpha}-1}{3 \bar{K}_{F \alpha}}$

Other variation coefficient is got by the characteristics of wind turbine gear transmission system.

The tooth root bending stress can be thought to obey logarithmic normal distribution, namely 
$\operatorname{In} \sigma_{F} \backsim N\left(u_{\text {In } \sigma_{F}}, S_{\text {In } \sigma_{F}}\right)$

(c)The bending fatigue strength calculation mechanical model

The bending fatigue strength calculation mechanical model is shown as follows.

$\sigma_{F G}=\sigma_{F \lim } Y_{S T} Y_{N T} Y_{\text {orelT }} Y_{\text {RrelI }} Y_{X}$

In (41), $\sigma_{F G}$ is calculation gear bending limit stress , $\sigma_{F l i m}$ is test gear name tooth root bending fatigue limit stress values, $Y_{S T}$ is test gear stress modification coefficient, $Y_{N T}$ is life factor, $Y_{\text {orelT }}$ is relative gear fillet sensitive coefficient, $Y_{\text {RrelT }}$ is relative tooth root surface condition coefficient , $Y_{X}$ is size coefficient.

(d) Gear bending fatigue strength distribution model

Gear bending fatigue strength is supposed to approximate normal probability distribution, logarithmic mean and logarithmic standard are calculated as follows.

$u_{I n \sigma_{F G}}=\operatorname{In} \bar{\sigma}_{F G}-\frac{1}{2} S_{I n \sigma_{F G}}^{2}$

$S_{I n \sigma_{F G}}^{2}=I n\left(1+\frac{S_{\sigma_{F G}}}{\bar{\sigma}_{F G}^{2}}\right)$

$S_{\sigma_{F G}}=C_{\sigma_{F G}} \bar{\sigma}_{F G}$

In (42) and (44)

$\bar{\sigma}_{F G}=\bar{\sigma}_{F \lim } \bar{Y}_{S T} \bar{Y}_{N T} \bar{Y}_{\text {סrelT }} \bar{Y}_{\text {RrelT }} \bar{Y}_{X}$

$C_{\sigma_{F G}}=\left(C_{\sigma_{F} \text { lim }}^{2}+C_{Y_{S T}}^{2}+C_{Y_{N T}}^{2}+C_{Y_{\tilde{S} e l T}}^{2}+C_{\text {RrelT }}^{2}+C_{Y_{X}}^{2}\right)$

The gear bending fatigue strength can be thought to obey logarithmic normal distribution, namely

$I n \sigma_{F G} \backsim N\left(u_{I n \sigma_{F G}}, S_{I n \sigma_{F G}}\right)$

3) Bearing failure unit reliability calculation model

The reliability of the planet bearing and high speed gear bearing is considered, every bearing failure is independent of each other. A large number of trials show the contact fatigue life of rolling bearings obeys Weibull distribution, the corresponding reliability assessment model is given as follows.

$R_{z}=\exp \left(-0.10536\left(\frac{t}{t_{90}}\right)^{q}\right)$

$t_{90}=\frac{16670}{n_{b}}\left(\frac{C_{r}}{f_{p} F}\right)^{\varepsilon}$

In (48) and (49), $C_{r}$ is basic rating load, $n_{b}$ is bearing work speed, $\varepsilon$ is life index, $t$ is bearing working life, $q$ is shape parameter.

\subsection{System reliability model}

To simplify the calculation, only the main aspects are considered, namely the load correlation and the use coefficient correlation. Assuming that gear and bearing failure is independent of each other, the gear reliability is calculated by the stress - strength interference theory and the numerical integral method.

$R_{\text {gear }}=\int_{-\infty}^{\infty} f\left(F_{t}\right) \int_{-\infty}^{\infty} f\left(K_{A}\right)\left[\prod_{i=1}^{l} \int_{-S_{i}\left(F_{t}, K_{A}\right)}^{\infty} f_{(}(r) d_{r}\right] d_{F_{t}} d_{K_{A}}$

The probability density function of $F_{\mathrm{t}}$ and $K_{A}$ respectively is the stress distribution of the failure mode.

According to (47) and (49), the gear reliability of low-speed, medium-speed and high-speed level bearings respectively is respectively $R_{g 1} 、 R_{g 2}$ and $R_{g 3}$, the bearing reliability of low-speed, medium-speed and high-speed level bearings respectively is respectively $R_{z 1} 、 R_{z 2}$ and $R_{z 3}$,so the reliability of whole gear transmission system is given as follows.

$R=R_{\mathrm{z} 1} R_{\mathrm{z} 2} R_{\mathrm{z3}} R_{g 1} R_{g 2} R_{g 3}$

\section{EXAMPLE ANALYSIS}

A 1.5 MW load-split wind power growth planetary gear concrete parameters is shown in Table I, low speed and medium speed level are respectively three planet uniform layout.

Table i model parameters

\begin{tabular}{|l|l|l|l|l|}
\hline & \multirow{2}{*}{ Low } & \multirow{2}{*}{ Medium } & High \\
\cline { 4 - 5 } & & & Input & Output \\
\hline teeth number & & & 109 & 30 \\
\hline sun teeth number & 24 & 25 & & \\
\hline planet number & 38 & 41 & & \\
\hline ring teeth number & 102 & 107 & & \\
\hline Modulus & 13 & 10 & 5.5 & 5.5 \\
\hline Pressure Angle & $20^{\circ}$ & $20^{\circ}$ & $20^{\circ}$ & $20^{\circ}$ \\
\hline Spiral Angle & $4^{\circ}$ & $4^{\circ}$ & $14.5^{\circ}$ & $14.5^{\circ}$ \\
\hline Tooth width(mm) & 345 & 175 & 145 & 145 \\
\hline
\end{tabular}

Wind turbine impeller diameter is $70.4 \mathrm{~m}$, design rotation speed is $14.93 \mathrm{r} / \mathrm{min}$, the average wind speed is $12.5 \mathrm{~m} / \mathrm{s}$, the wind density is $1.225 \mathrm{~kg} / \mathrm{m} 3$, wind energy utilization coefficient is 0.32 .

The wind gear increaser external wind input load is shown as Fig. 4. 


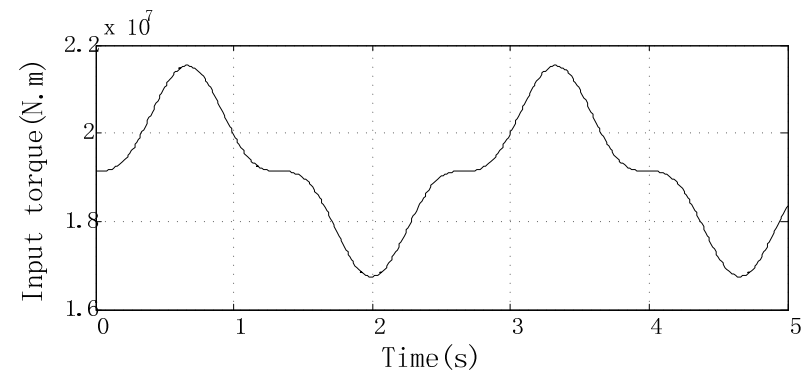

Figure 4. External wind input load

The dynamic meshing force time domain response of low and medium speed level is shown in Fig. 5.
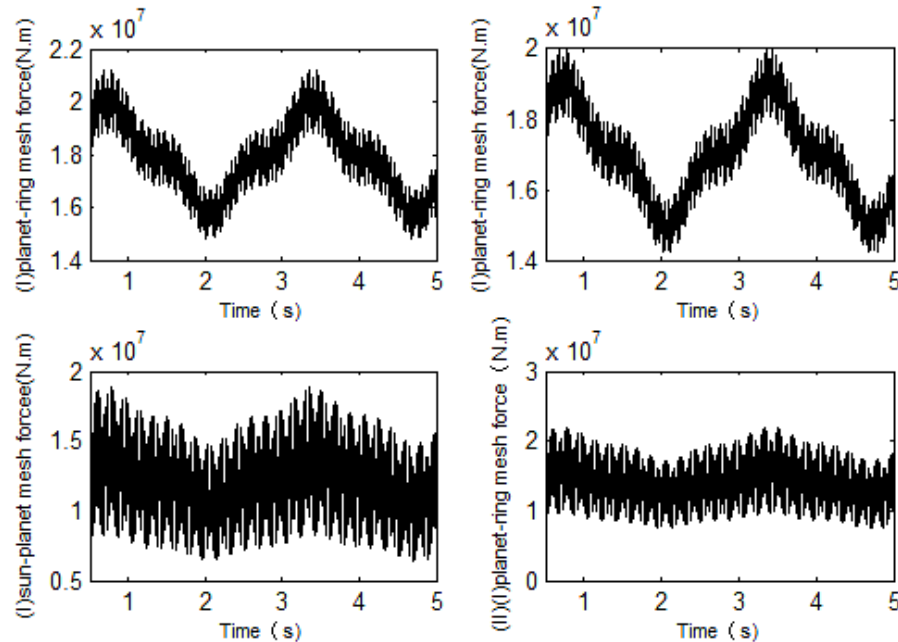

Figure 5. Dynamic meshing force

According to (1)- (51), the corresponding program is wrote. Under the condition of external wind input load and dynamic meshing force time domain response, the entire transmission system reliability based on dynamic analysis is 0.892 .

\section{CONCLUSION}

On the basis of the dynamics model analysis, the load-split wind power growth planetary gear train reliability evaluation model is obtained under random wind speed, the reliability of load-split wind power growth planetary gear train is obtained. It can lay the foundation for wind turbine gear transmission system optimization design.

\section{ACKNOWLEDGMENT}

In this paper, the research was sponsored by Chongqing Municipal Education Commission of Science and Technology Research Project (Project No. KJ132205).

\section{REFERENCES}

[1] Li chun-ling , liu bo, xujie, an zong-wen, "Dynamic reliability analysis of sun-wheel in gearbox of wind turbine", Journal of Lanzhou University of Technology, 2013,39(5):28-31.

[2] Zhao ying, "High-power wind power gear box design and reaearch”,Jilin university master thesis,2012.

[3] MUSIAL W, BUTTERFIELD S, MCNIFF B. "Improving wind turbine gearbox reliability”, European Wind Energy Conference, May 7-10, 2007, Milan, Italy. Springfield:U.S Department of Commerce National Technical Information Service, 2007:1-13.

[4] Zhang xuefeng,"Wind blades still need policies Suggestions to promote the development of wind power industry in our country”, China electric power enterprise management,2009(4):44-45.

[5] Rao S S. "Reliability analysis and design of epicyclic gear trains”, Journal of Mechanical D esign, ASME, 1979 ,101 (3): 625-632.

[6] $\mathrm{Hu}$ qingchun,duan fuhai, wu shangsheng,"Research on Reliability of Closed Planetary Transmission Systems”, China Mechanical Engineering, 2007,18(2):146-149.

[7] Wu shang-sheng,duan fu-hai,hu qing-chun,"The influence of allocation of systematic parameters on the reliability of multi-staged planet gear transmission”,Journal of Machine Design,2007, 24(10): 43-46.

[8] Li chun-ling, Liu bo ,xu jie, an zong-wen, "Dynamic reliability analysis of sun-wheel in gearbox of wind turbine”,Journal of Lanzhou University of Technology,2013,05,28-31.

[9] JungangWang, Yong Wang, and Zhipu Huo, “Analysis of Dynamic Behavior of Multiple-Stage Planetary Gear Train Used in Wind Driven Generator”, The Scientific World Journal, Volume 2014, Article ID 627045, 11 pages.

[10] MAATAR M, VELEX P, “An analytical expression for the time-varying contact length in perfect cylindrical gears: Some possible applications in gear dynamics", Journal of Mechanical Design, 1996, 118(12): 586-589.

[11]Yang xiaoyu, "Finite element analysis and experiment research on dynamic charater of gear system”, Changchun Institute of optics and fine mechanics and physics,2005,1315.

[12]Jiang kun, "Dynamic charateristics of parallel shaft gear rotor system”, Northeastern University, 2009, 29-37.

[13]Gear manual editorial board, Gear manual, Mechanical Industry Press, 2006 